\section{A concept of perceived orientation}

\author{
NAUM YAKIMOFF and LEON MITRANI \\ Institute of Physiology \\ Bulgarian Academy of Sciences \\ Sofia 1113, Bulgaria
}

A great number of investigations have been made in an attempt to clarify and understand the visual perception of form, size, and location. Some neurophysiological models have been proposed to explain the basic experimental facts (Glezer, Doodkin, Cooperman, Levshina, Nevskaya, Podvigin, \& Prazdnikova, 1975; Lindsay \& Norman, 1972).

The ability of man to assign orientation to objects has also drawn some attention, mainly in connection with the perception of form. A monograph has been published on this matter (Rock, 1973) which presents an excellent review of the studies made and the personal standpoint of the author. The essence of this standpoint seems to be that "the implicit cognitive description of a figure is a function of the figure's directions: whether a figure's long axis is 'vertical' or 'horizontal,' whether the figure rests on a base or stands on a point, whether it is symmetrical or not, and so forth."

However astonishing it may be, investigators usually are not concerned with the proper notion of the term "orientation." It is generally accepted that everybody knows what orientation is and therefore no special definition or more detailed discussion is needed. We feel that some attention must be paid to what we call perceived orientation and to the connection of our perception to the geometrical or other features of the objects. A clarification is also needed if we want to give a neurophysiological explanation of the findings or even to suggest some simple models.

Let us stipulate, for discussion, only real twodimensional objects-figures, drawings, etc; visually perceived. What is the meaning of the questions: "What is the orientation of a contour drawing?" "What is the orientation of a combination of light and dark spots?" "What is the orientation of a cluster of dots?"

If we have a line segment drawn on a sheet of paper, we may assume that the orientation of this simple object is given by the angle the segment forms with one of the existing real or imaginary lines, i.e., the edges of the sheet, the horizontal or the vertical line, some other lines drawn on the sheet, etc. In all cases, when we speak of perceived orientation of a line segment, it suffices to accept a convention for a real or imaginary line of reference and a direction for measurement of angles.
The question of "orientation" for two-dimensional objects is far more complicated. Some conventions corresponding to our perception of simple figures might be accepted. The orientation of a rectangle may well be described by the angle between one of its longer sides and some other line. The orientation of an ellipse may be the corresponding angle for its longer axis. Such definitions will be in accordance with our perception of each figure. It is meaningless to assign orientation to some figures with more than one axis of symmetry. What should be the orientation of a circle, a square, or of an Archimede's spiral?

It seems clear that one may speak of perceived orientation of a figure only if there is a real or imaginary line segment defined by the figure that is perceived either as longer than all other such segments or as corresponding to some other salient principle. The essential point is that this segment should be unique. When there are more than one such segments (or lines) corresponding to the same principle, the assignment of orientation becomes ambiguous, difficult, and even impossible.

\section{Orientation as Optimization}

The human visual system is so organized that it is influenced essentially by luminance differences in the visual field. A uniform visual field is meaningless for the visual system. It seems also that our vision is oriented to some saliencies in the pattern. The underlying of contours is, in fact, a deduction of the maximal luminance gradient. Our line of sight is usually oriented toward the brightest point, the point with maximum curvature, etc. There is a chance that the perception of the orientation of a two-dimensional figure may also be a result of some optimization process in the visual system aimed at determining a line, or a segment, real or imaginary.

From a purely geometrical standpoint, it is impossible to say what kind of optimization takes place to single out one given line or segment that may represent the perceived orientation of a figure. There are many ways to determine salient lines in complex patterns. Even in such a simple figure as the rectangle, it is not clear why the orientation is given by the longer sides instead of the still longer diagonals. Obviously the optimization is related to some other specific characteristics rather than to relative dimensions alone.

It is clear that we can answer the question about the kind of optimization that takes place only by experimentation. We must take a figure to which it is possible to assign several different optimum lines and to find out experimentally which of these lines determines the perceived orientation. If we find, 
for different figures, that the perceived orientation is always connected to lines of the same kind (defined by the same process of optimization), we may be able to give a general definition of the term "orientation" based on a specific process of optimization that goes on in our visual system.

Some optimal lines should be eliminated beforehand. These are the lines which are not invariant in respect to rotation of the figure. We must deal only with lines (directions) steadily connected with the figure. In all probability, it is in this sense that the term "intrinsic orientation" is used. For example, the line of regression drawn upon a cluster of experimental points depends on the choice of the coordinate system and therefore does not represent the cluster itself, but its connection with the coordinates.

Nevertheless, there are many (it is not even possible to know how many) different ways of obtaining optimum lines connected with a figure. For example, there are: the line connecting the most distant points of the figure; the line for which the sum (the integral) of the distances to each point of the figure is minimal or maximal; the line that divides the figure into two parts equal in luminance, homogeneity, area (or some other factor); the line for which the sum or the integral of the squared distances to each point of the figure multiplied to by the luminance is minimal or maximal, etc. There is no need to say that perceived orientation of known figures is determined by the whole previous experience. The drawing of a table or of a stool may induce perception of a given orientation regardless of the specific dimensions of the figure only because we know that tables and stools more often stand on their feet rather than upside down. It is the same for drawings of animals, houses, etc. Only men who are not accustomed to the use or the usual posture of the objects may ascribe an orientation that is entirely determined by the shape, dimensions, or other specific characteristics.

Therefore if we want to know how man perceives orientation-intrinsic orientation-it is better to use forms or objects that are not familiar to the subjects and to test only invariant directions (lines) connected with these objects. In this paper, we give as an example some of our experiments and results in this field. Elongated dot patterns were used in an assignment of orientation task. Although these kinds of stimuli do not contain any lines or contours, they are usually perceived as a whole pattern.

\section{METHOD}

\section{Apparatus and Stimuli}

The subject was seated in a darkened room in front of a circular matt screen, $50 \mathrm{~cm}$ in diameter and at a distance of $75 \mathrm{~cm}$ (36.9 $9^{\circ}$ of visual angle). A slide projector was used to present stimuli on the screen. These were the five different dot patterns shown in Figure 1.

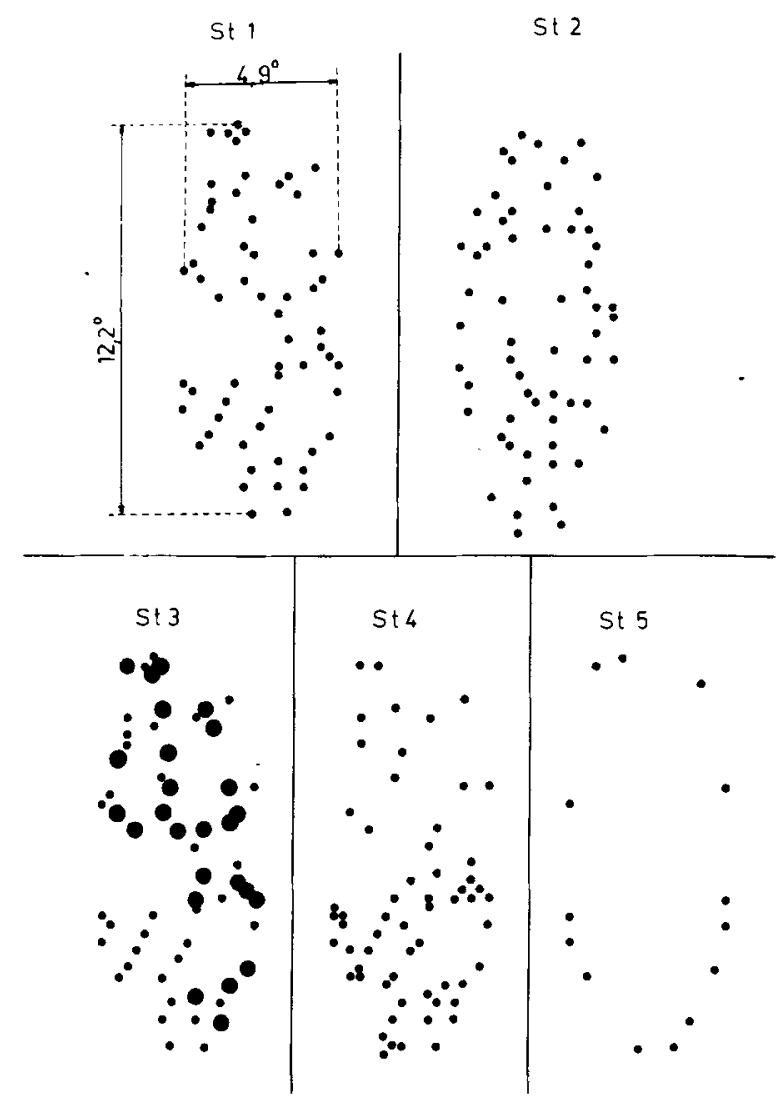

Figure 1. The five dot patterns used in the experiment.

Stimulus 1 (St 1) and Stimulus 2 (St 2) consisted of 60 equal bright dots (the negatives are given in the figure). The two stimuli differed in the distribution of the dots inside an ellipse with a $5 / 2$ ratio between its main axes. A random-number table was used in both cases to determine the position of each point. The dots in Stimulus 3 (St 3) had the same distribution as in St 1 , but some were enlarged to subtend an area nine times their initial area. Stimulus 4 (St 4) was derived from $\mathrm{St} 1$ by a transposition of part of the dots from one half to the other. Stimulus 5 (St 5 ) consisted of the dots that form the apices and lie on the sides of the polygon that embraced all the dots of St 1 .

All stimuli subtended an area of $12.2^{\circ} \times 4.9^{\circ}$ of visual angle. The dots of St 1, St 2, St 4, and St 5 and the small dots of St 3 each subtended $.1^{\circ}$ of visual angle. The large dots of St 3 subtended $.3^{\circ}$ of visual angle.

\section{Procedure}

The stimuli were presented in succession according to their number given in Figure 1. Their orientation on the screen was randomly chosen, except that the longer axis of the imaginary determining ellipse was never to be close to a vertical or horizontal position (in Figures 1 and 2, the longer axes for all patterns are given vertically only for convenience). The subject's task was "to determine the orientation of the dot pattern" by means of an adjustable bright line projected on the screen with a length subtending $26.3^{\circ}$ and a width subtending $.1^{\circ}$ of visual angle. Every subject made 10 adjustments of the line, i.e., twice for each stimulus. A total of 100 estimates for each stimulus was obtained from the experiment.

After each trial, a picture was taken of the stimulus and the line of estimation. Two light targets, invisible to the subject, marked 

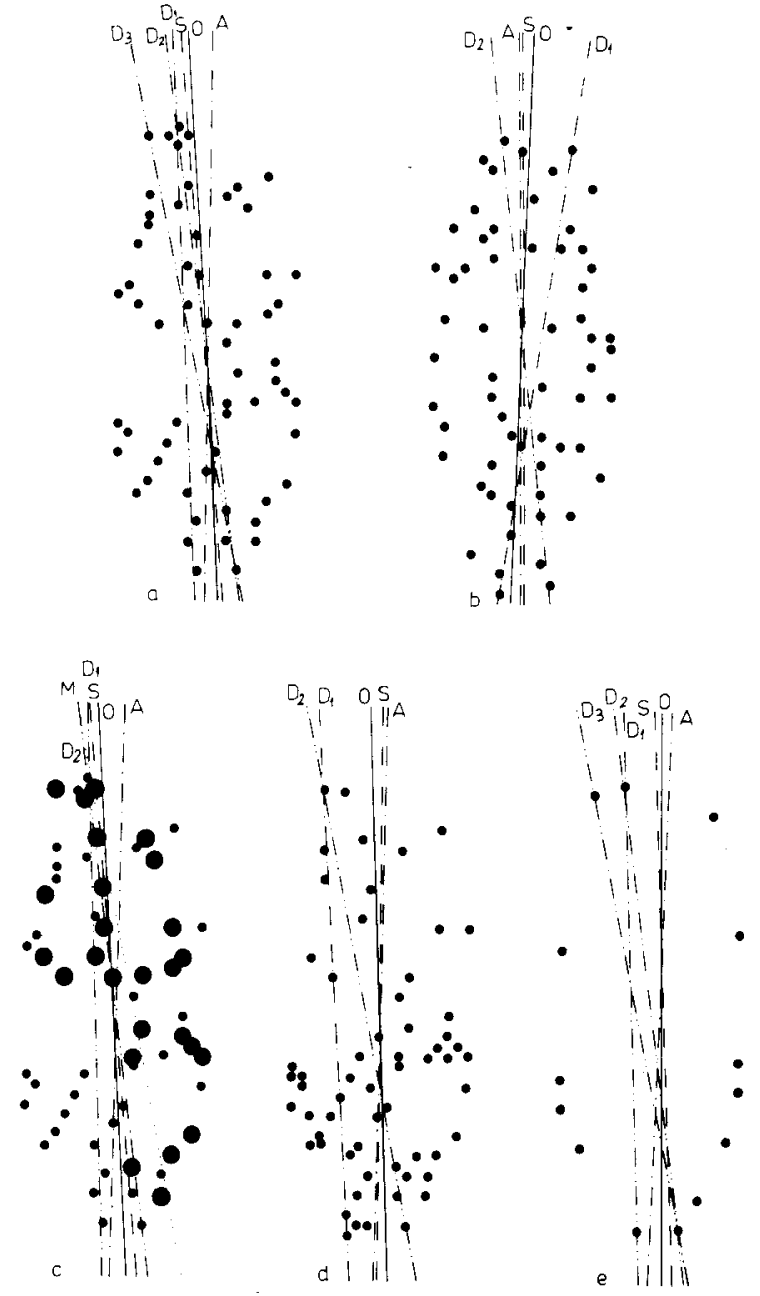

Figure 2. Different optimum lines as well as the line of perceived orientation superimposed on the five test stimuli (a, $b$, c, d, and e) $\longrightarrow$, lines of perceived orientation; - $\ldots$ - $-\mathrm{S}$, lines corresponding to the minimal sum of the squared distances between each point of the stimulus and this line; $-\cdots \ldots$ A, the axis of the determining ellipse; $-\ldots \ldots$, lines drawn between the most distant points of the stimuli: and $M$, line (axis) of the least moment of inertia.

the position of the horizontal diameter of the screen. Thus we were able to find the equation of each line of estimation in a previously fixed coordinate system as well as the orientation of this coordinate system in respect to the horizontal diameter of the screen.

\section{Subjects}

The subjects were 50 volunteers, men and women between the ages of 25 and 45 years, with higher or secondary education from the scientific and aid staff of the Institute. All had normal or corrected vision.

\section{RESULTS AND DISCUSSION}

The statistical analysis of the data showed that the mean estimated orientation of all patterns used does not depend on the orientation of the longer axis of the determining ellipse in respect to the horizontal diameter of the screen $(p<.05)$.

The most remarkable result emerges from the strategy adopted by the subjects to adjust the line of estimation. Of the 50 subjects tested, 49 performed the task by adjusting the line so that it crossed the dot pattern. The average lines of perceived orientation (O) are presented in Figure 2 ( $a, b, c, d$, and e). Only one of the subjects in all 10 presentations made his adjustment with the line outside the dots but in alignment with the mean estimated orientation given by the other subjects. This result strongly supports the suggestion that the perceived orientation of our stimuli is determined by the orientation of a definite line characteristic for the set of dots and singled out by the visual system.

Several different optimum lines, invariant in respect to rotation of the stimuli, were tested in an attempt to match the experimental lines of orientation. These optimum lines, shown in Figure 2, are: the lines (D) drawn between the most distant points of the stimuli; the long axis (A) of the determining ellipse; and the lines (S) for which the sum of the squared distances from each point of a stimulus to this line is minimal. All these lines might be regarded as geometrically determined characteristics of the stimuli. The choice of St 3 permitted us to test another optimum line (M), the axis about which this stimulus should have a minimal moment of inertia if considered as a system of material points, the mass of each point being proportional to its area.

It appeared that of all lines tested, only line $S$ can be assumed to match the line of perceived orientation in all cases. Only this line lies almost entirely within the $95 \%$ confidence interval of the average line of perceived orientation for each stimulus. It is true that, for St 2 and St 4, line A can also be assumed to match the average orientation line. In these cases, lines $S$ and $\mathrm{A}$ are, in fact, very close. However, when the distribution of the dots determines a difference between lines $\mathrm{S}$ and $\mathrm{A}$, as in St 1 and St 5 , it is only line S that matches reliably the line of perceived orientation.

\section{CONCLUSIONS}

The results of this experiment, although obtained with a restricted number of dot patterns with similarly shaped envelopes and even similar dot densities (except for St 5), might be regarded as support of our initial idea that a definite process of optimization in the visual system underlies the perception of orientation. We were able to demonstrate that for the five stimuli used there exists an optimum line that matches the experimentally determined line of orientation. This line is determined by the distribution of the centers of the dots in each pattern and corresponds to a common optimization process: a minimization of the squared distances between each dot of the given pattern and this line. 
This experiment is no more than an example that supports the possibility for elaboration of a general definition of the intrinsic orientation of two-dimensional objects. No doubt a great number of experiments are needed with a variety of stimuli and experimental conditions before our specific claim can be confirmed. Further investigations are in process in our laboratory.

\section{REFERENCES}

Glezer, V. D., Doodkin, K. N., Cooperman, A. M., Leushina, L. I., Nevskaya, A. A., Podvigin, N. F., \& Prazdnikova,
N. V. Visual recognition and its neurophysiological mechanisms. Leningrad: Nauka, 1975. (In Russian)

Lindsay, P. H., \& Norman, D. A. Human information processing. An introduction to psychology. New York: Academic Press, 1972.

Rock, I. Orientation and form. New York: Academic Press, 1973. 